\title{
Delivery of Suramin as an Antiviral Agent through Liposomal Systems
}

\author{
Eloise Mastrangelo, ${ }^{[\mathrm{a}, \mathrm{b}]}$ Stefania Mazzitelli, ${ }^{[\mathrm{c}]}$ Jacopo Fabbri, ${ }^{[\mathrm{c}]}$ Jacques Rohayem, ${ }^{[\mathrm{d}, \mathrm{e}]}$ \\ Janne Ruokolainen, ${ }^{[f]}$ Antti Nykänen, ${ }^{[f]}$ Mario Milani, ${ }^{[a, b]}$ Margherita Pezzullo, ${ }^{[a, b]}$ \\ Claudio Nastruzzi, ${ }^{*[c]}$ and Martino Bolognesi ${ }^{*[a, b]}$
}

\begin{abstract}
Norovirus RNA-dependent RNA polymerase (RdRp) is a promising target enzyme for the development of new antiviral drugs. Starting from the crystal structure of norovirus RdRp, we had previously performed an in silico docking search using a library of low-molecular-weight compounds that enabled us to select molecules with predicted enzyme inhibitory activity. Among these, the polysulfonated naphthylurea suramin proved to inhibit in vitro both murine and human norovirus polymerases,
\end{abstract}

with $\mathrm{IC}_{50}$ values in the low micromolar range. The negatively charged inhibitor, however, displayed poor cell permeability in cell-based experiments. Therefore, we produced different suramin-loaded liposome formulations and evaluated their activities in cell-based assays using murine norovirus cultivated in RAW 264.7 macrophages, as a model for norovirus genus. The results obtained show that suramin, when delivered through liposomes, can effectively inhibit murine norovirus replication.

\section{Introduction}

The genus Norovirus consists of genetically diverse singlestranded RNA nonenveloped viruses, in the Caliciviridae family. Noroviruses are the most common cause of viral gastroenteritis in humans and affect around 267 million people annually, causing over 200000 deaths each year ${ }^{[1]}$ Although the relative proportion of mortalities associated with noroviruses is considered to be very low, several other serious conditions, such as necrotising enterocolitis (NEC) in neonates ${ }^{[2]}$ and seizures, ${ }^{[3]}$ as well as an active role for noroviruses in diarrhoea-related deaths in the developing world, has been suggested. ${ }^{[4]}$ Since no effective vaccines/antiviral treatments are available, there is an urgent need for antiviral drugs able to contain the outbreaks of this highly transmissible virus. The norovirus genome contains three open reading frames (ORF1-3). ORF1 is translat-

[a] Dr. E. Mastrangelo, ${ }^{+}$Dr. M. Milani, Dr. M. Pezzullo, Prof. M. Bolognesi Department of Biosciences \& CIMAINA, University of Milano

Via Celoria 26, 20133 Milano (Italy)

E-mail: martino.bolognesi@unimi.it

[b] Dr. E. Mastrangelo, ${ }^{+}$Dr. M. Milani, Dr. M. Pezzullo, Prof. M. Bolognesi Biophysics Institute (CNR-IBF)

Via Celoria 26, 20133 Milano (Italy)

[c] Dr. S. Mazzitelli, J. Fabbri, Prof. C. Nastruzzi

Department of Life Sciences \& Biotechnology, University of Ferrara Via Fossato di Mortara 17/19, 44100 Ferrara (Italy)

E-mail:nas@unife.it

[d] Dr. J. Rohayem

Institute of Virology, Dresden University of Technology

Fiedlerstrasse 42, 01307 Dresden (Germany)

[e] Dr. J. Rohayem

Riboxx $\mathrm{GmbH}$

Pharmapark Radebeul, Meissner Strasse 191, 01445 Radebeul (Germany)

[f] Prof. J. Ruokolainen, Dr. A. Nykänen

Department of Applied Physics, Aalto University

Puumiehenkuja 2, 02150 Espoo (Finland)

$\left.{ }^{[}\right]$These authors contributed equally to this study. ed into a large poly-protein precursor, cleaved into six nonstructural (NS) proteins. ${ }^{[5]}$ Among the NS proteins, NS7 is endowed with RNA-dependent RNA polymerase (RdRp) activity, which plays a key role in genome replication as well as in the synthesis and amplification of subgenomic RNA. ${ }^{[6]}$

Starting from the crystal structure of Norovirus RdRp, we previously explored protein sites for ligand binding, screening in silico a library of small molecules in order to identify commercially available compounds endowed with predicted high affinity for the selected enzyme region ${ }^{[7]}$ Through such docking procedures, among other compounds, we identified suramin, a polysulfonated naphthylurea that has been used as the drug of choice for treatment of African trypanosomiasis and onchocerciasis $^{[8]}$ since 1924 . Suramin proved to strongly inhibit new RNA production by both murine and human Norovirus RdRps in vitro, with $\mathrm{IC}_{50}$ values in the low micromolar range. The discovery of new activities for suramin has recently renewed interest in this drug as a potential antiviral agent, as well as for the treatment of different forms of cancer, including renal, breast and ovarian cancer. Unfortunately, suramin treatment is associated with severe adverse side effects, such as neurotoxicity, adrenal insufficiency, anemia, lymphopenia, and coagulopathy. ${ }^{[9,10]}$ In addition, suramin is a rather complex, highly charged drug hosting six sulfonate groups; all these are intrinsic features that confer suramin with very low membrane permeability and limited cell internalization. ${ }^{[11]}$ In order to overcome such major hurdles, a preformulation study was carried out with the aim of developing liposomal formulations for suramin delivery.

Over the years, liposomes have attracted considerable attention as delivery systems for antitumor drugs, antiviral agents, and for gene delivery protocols. In particular, cationic liposomes have recently emerged as leading nonviral vectors in 
worldwide gene therapy clinical trials. ${ }^{[12]}$ Cationic liposomes are generally composed of neutral and cationic lipids; the resulting positively charged liposomes have been shown to acquire/ entrap (negatively charged) nucleic acids. Such complexes are able to transfect nucleic acids (DNA and RNA) in cells, resulting in the expression of the protein encoded in a DNA plasmid within the target cells. ${ }^{[13]}$ Similarly to the nucleic acids, the negatively charged suramin might be bound by cationic liposomes in order to develop formulations, exhibiting decreased drug-related toxicity, enhanced cellular uptake, and possible higher accumulation in macrophage-rich organs. ${ }^{[14]}$ An additional aspect relating to liposomes is their ability to deliver their cargo directly to the early endosome, with further processing in the later endosome and lysosome, hence mimicking the route used by RNA viruses, such as noroviruses, to deliver its genomic RNA to the targeted cell. Therefore, when using liposomal formulations to deliver suramin into the cells, a facilitated interaction within the endosomal compartment between genomic RNA and the drug might occur, with subsequent increased potency of the drug.

Numerous techniques have been developed to obtain liposomal preparations, including thin-film hydration, ${ }^{[15]}$ organicsolvent injection, ${ }^{[16]}$ reverse-phase evaporation, ${ }^{[17]}$ and dehydration-rehydration. ${ }^{[18]}$ Most of the described preparation techniques are unfortunately not suitable for large-scale manufacturing due to low batch reproducibility and predictability. ${ }^{[19]}$ For example, the thin-film hydration technique, which represents the most widespread method for preparing liposomes on a laboratory scale, yields heterogeneous multilamellar vesicles (MLV) over $1 \mu \mathrm{m}$ in diameter, requiring further preparation steps (i.e., extrusion) to obtain homogeneous vesicles.

In the current study, cationic liposomes entrapping suramin were produced by an ethanol injection method. ${ }^{[20]}$ This particular technique, which is rather simple to implement, uses ethanol as a co-solvent and does not require homogenization devices; moreover, the experimental approach can be easily scaled up for industrial application. Liposome formation occurs due to the miscibility of ethanol and water, resulting in the diffusion of the ethanol molecules to the aqueous solution. We explored the roles of different experimental parameters on the main suramin-loaded liposome characteristics, such as mean vesicle size, the vesicle size distribution and polydispersion. In addition, morphology of the suramin-loaded liposomes obtained was determined by cryogenic transmission electron microscopy (cryo-TEM) and atomic force microscopy. A selected number of liposomal formulations was then tested for cytotoxicity and anti-norovirus activity in cell-based assays. We demonstrate that suramin, when delivered through liposomes, can effectively inhibit murine norovirus replication with $\mathrm{EC}_{50}$ values in the micromolar range.

\section{Results and Discussion}

\section{General considerations}

To overcome limitations associated with many basic liposome preparation techniques, including the difficulties met in pilot- ing and scaling up to the industrial production, the ethanol injection method appeared to be the optimal choice. In general, ethanol injection and its variations involve the solution of the chosen lipids in ethanol (or occasionally other short-chain alcohols, such as methanol or isopropanol), followed by rapid transfer of the lipid solution into water. The addition of lipids to the water phase, at a temperature above the melting temperature $\left(T_{m}\right)$, leads to the self-assembly of lipids into liposomes. The main advantage of the ethanol injection method resides in the formation, in just one step, of liposomes endowed with a quite narrow size distribution, without degradation or oxidation of lipids or drugs.

Despite the easily applied protocol, further improvements to the ethanol injection method are needed to optimize the general pharmaceutical characteristics of the produced vesicles, including size uniformity, encapsulation efficacy, and reproducibility/predictability from batch to batch. To this aim, in the current paper, the classic ethanol injection protocol (referred to as "by hand") was indeed comparatively analyzed relative to the "controlled" injection method in which the phosphatidylcholine (PC)/ethanol solution is injected into the water phase in a controlled fashion, via a syringe pump, allowing precise control of ethanol diffusion in water. The mixing of ethanol with water is indeed the crucial step leading to liposome assembly since the migration of alcohol molecules, originally surrounding the lipid molecules, away from the lipids into the bulk, drives liposome formation. Comparison of the physicochemical and activity properties of liposomes produced by hand versus controlled injection method appears particularly interesting. The majority, if not all, of the papers describing the preparation of liposomes by ethanol injection method refers indeed to hand injection procedures, which lack the reproducibility and reliability criteria needed for industrial scale up.

\section{Preparation of liposome containing suramin}

Depending on drug solubility and polarity characteristics, drug molecules can be encapsulated in liposomes following several different approaches. Highly water-soluble drugs are generally spontaneously entrapped into the aqueous internal cavity of liposomes. However, the efficiency of encapsulation, especially in the case of ethanol injection, can be quite low; thus, the encapsulation of hydrophilic drugs into liposomes presents unique challenges in the liposome design stage.

Suramin is a symmetric polyanionic naphthylurea, highly soluble in water (up to $50 \mathrm{mg} \mathrm{mL}^{-1}$, resulting in the formation of a clear, faint yellow solution) but only sparingly soluble in $95 \%$ ethanol. Due to such low ethanol solubility, suramin was initially dissolved in water and later electrostatically complemented with L-lysine, The resulting solutions were mixed with a PC/ethanol solution containing dimethyl-dioctadecylammonium bromide (DDAB) and injected "by hand" or by syringe pump ("controlled") into an external water phase. In the case of the controlled preparation method, the ethanol injection velocity was controlled by means of a syringe pump and kept at a constant rate of $500 \mu \mathrm{L} \mathrm{min}^{-1}$. According to the literature, ${ }^{[20]}$ the injection rate has no significant effect on the mean vesicle 
size, due to the rapid (almost instantaneous) diffusion of the solvent within the aqueous phase.

The effects of phospholipid concentration on cationic liposome size were firstly investigated both through "by hand" and "controlled" methods; the respective photon correlation spectroscopy (PCS) measurements are reported in Tables 1 and 2, and Figures 1 and 2. For the analyzed phospholipid con-

\section{Cryo-TEM and atomic force microscopy liposome characteri- zation}

Since the physicochemical properties and performance of liposomes (i.e., drug release or cell uptake) are deeply affected by various characteristics, such as the size, shape, and number of lamellae, an accurate morphological characterization of the liposomes obtained is mandatory before any biological evaluation. A single method for size characterization of liposomes is not capable of determining size distributions with adequate accuracy and reliability. In this respect, results of the PCS measurements were compared to those provided by cryo-TEM and atomic force microscopy (AFM) analyses. The TEM technique enables the imaging of liposome architecture, to study their morphology and lamellar shapes together with an estimate of liposome size. The negative-stain TEM images demonstrate that both empty (Figure $4 \mathrm{~A}$ ), or suraminloaded (Figure 4B) liposomes, obtained by the "controlled" ethanol injection, were spherical in shape. The majority of the empty liposomes had a size smaller than $100 \mathrm{~nm}$ in diameter, in agreement with the PCS analysis. In contrast, liposomes containing suramin were comparatively less homogenous and bigger in size. In the case of the AFM analysis, the data also proved to be in agreement with the PCS results, taking into consideration that in the AFM measurements the liposomes are adsorbed on a solid surface, while for the PCS analyses the lipocentrations ( 3 and $9 \mathrm{~mm}$, final concentration), a size increase from $26.0 \pm 2.5$ to $150.0 \pm 12.0 \mathrm{~nm}$, and $11.5 \pm 0.3$ to $136.2 \pm$ $5.7 \mathrm{~nm}$, was observed in liposomes prepared "by hand" (VEI-h) and "controlled" methods (VEI-c), respectively. In addition, when suramin was loaded into liposomes, a more significant increase in the liposome size was detected, due to the electrostatic interaction of the drug molecule with the cationic liposomes, yielding larger cationic complexes. Notably, by a comparative analysis of both preparation methods, the formation of unilamellar vesicles with a small diameter and a low polydispersion index (PDI) was achieved only through the "controlled" method (see Tables 1 and 2, and Figure 3). somes are in suspension. As expected, after deposition, liposomes showed a progressive tendency to turn into a flattened and less symmetric structure, described as planar vesicles.

\section{Encapsulation efficacy}

The elution profile of liposomes containing suramin, obtained after sepharose $4 B$ chromatography, is shown in Figure 5. The elution profile shows two well-resolved peaks. The major peak (indicated by the solid arrow) contains suramin-loaded liposomes; the second broad peak reflects the elution of free suramin (indicated by the open arrow). The presence of suramin in both peaks was shown by recording the UV spectrum of the 
A)
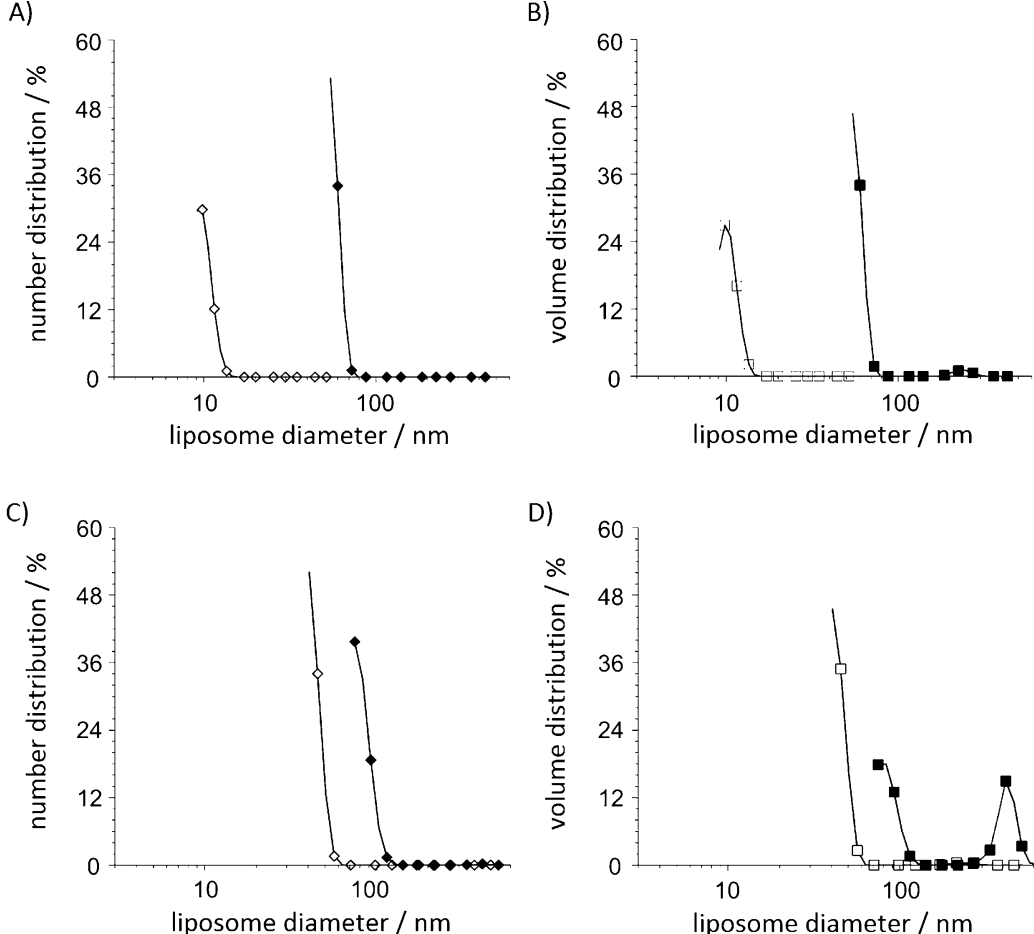

D)

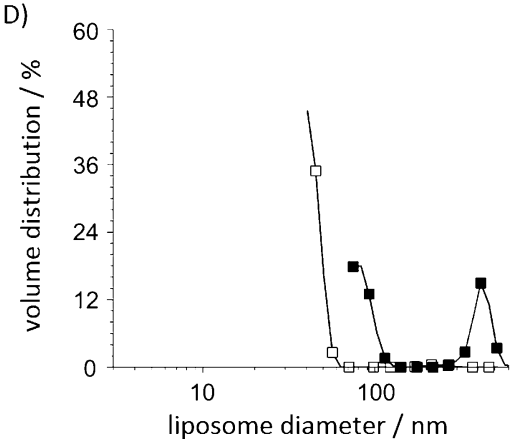

Figure 1. Size distribution plot by number (panels $A$ and $C$, diamonds) and by volume (panels $B$ and $D$, squares) of empty (open symbols) or suramin-loaded liposomes (filled symbols). Liposomes were prepared by the ethanol injection method, following the procedure referred to as "by hand" employing phosphatidylcholine (PC) at the final concentration of $3 \mathrm{~mm}$ (panels $A$ and $B$ ) or $9 \mathrm{~mm}$ (panels $C$ and D). For the complete description of the preparation procedure and liposome composition, see the Experimental section.
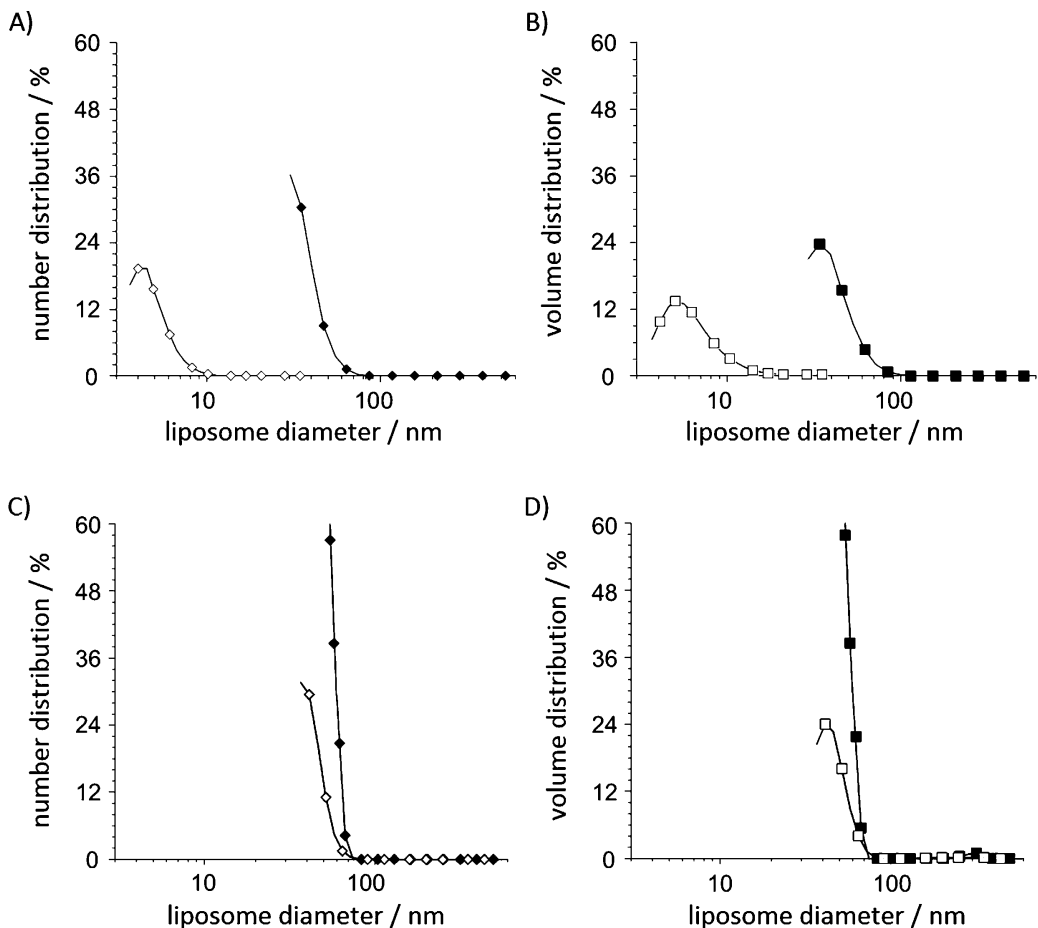

Figure 2. Size distribution plot by number (panels $A$ and $C$, diamonds) and by volume (panels B and D, squares) of empty (open symbols) or suramin-loaded liposomes (filled symbols). Liposomes were prepared by the ethanol injection method following the procedure referred to as "controlled" employing phosphatidylcholine (PC) at the final concentration of $3 \mathrm{~mm}$ (panels $A$ and $B$ ) or $9 \mathrm{~mm}$ (panels $C$ and D). For the complete description of the preparation procedure and liposome composition, see the Experimental section.

corresponding fractions-the absorption maximum of suramin is at $318 \mathrm{~nm}$. The suramin entrapment yield was estimated by comparing the absorption of the isolated liposomes at $318 \mathrm{~nm}$ with suramin solutions of known concentrations. The yield of entrapment for liposomes prepared with $3 \mathrm{~mm}$ PC was $72.8 \pm 9.3 \%$ and $88.5 \pm 11.1 \%$ for VEl-h (suramin) and VEl-c (suramin) liposomes, respectively. Such results indicate the high liposome entrapment efficacy achieved when suramin was delivered through the "controlled" method.

\section{Cell-based assays}

Murine norovirus (mNV) is readily propagated in RAW 264.7 murine macrophage cells. Hence, mNV is an interesting model for the norovirus genus as to pathogenicity, multiplication cycle, and immunity studies that may also reflect properties of other norovirus genotypes. The potential inhibitory effects of suramin-loaded liposomal formulations on the in vitro replication of $\mathrm{mNV}$ were evaluated using a cell proliferation assay, as previously described. ${ }^{[21]}$ In particular, the suramin-loaded liposomes complexed with L-lysine, and obtained by the "controlled" method, were chosen and compared with the suramin/lysine salt alone. As an alternative approach for the delivery of suramin, polymeric micelles were also considered.

Such formulations are indeed a class of nanoparticles with a core-shell structure that form spontaneously by self-assembly of amphiphilic block co-polymer unimers in water. Similarly to liposomes, polymeric micelles could result in some advantages over conventional formulations, including controlled delivery, alteration of the drug circulation time, changes in the cellular dis- 
A)
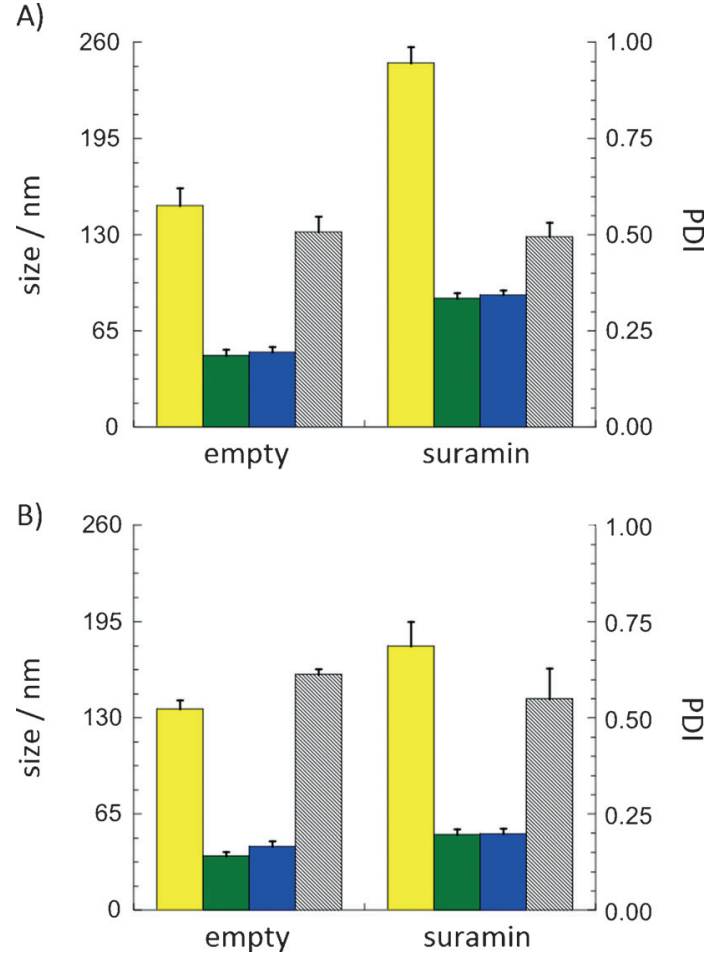

Figure 3. Comparative analysis of the dimensional characteristics of empty and suramin-loaded liposomes prepared by the procedures referred as "by hand" (panel A) and "controlled" (panel B) employing phosphatidylcholine (PC) at a final concentration of $9 \mathrm{~mm}$. Data refer to Z-average (yellow), mean by number (green), mean by volume (blue) and to the polydispersity index (stripes).
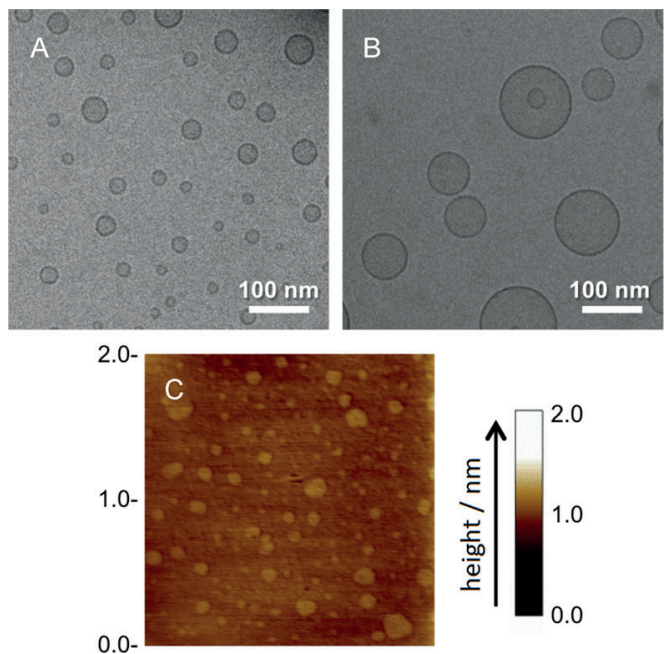

Figure 4. Morphological and dimensional characterization of suramin-loaded liposomes by B) cryogenic transmission electron microscopy (cryo-TEM) and C) atomic force microscopy (AFM). For comparison, the cryo-TEM analysis of empty liposomes is also reported in panel A. The order of magnification is indicated by the relative bars.

tribution, and increase the amount of drug delivered to target cells. As a result, suramin delivered by liposomes inhibited the replication of the virus. In particular, in a virus yield reduction assay performed in RAW 264.7 cell cultures, the $\mathrm{EC}_{50}$ value for inhibition of viral progeny formation was $0.3 \mu \mathrm{m}$ (Table 3), with
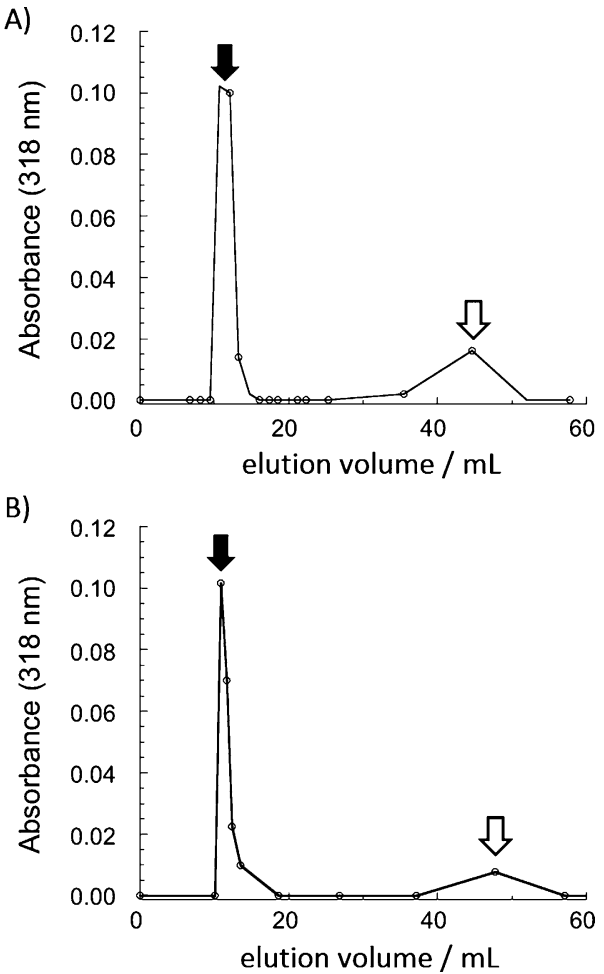

Figure 5. Elution profile of suramin-loaded liposomes on sepharose $4 \mathrm{~B}$ gel-

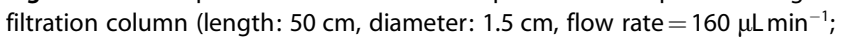
$3.2 \mathrm{~mL} /$ fraction). The VEI-h (suramin) and VEI-c (suramin) were prepared by the ethanol injection method following the procedure referred to as A) "by hand" or B) "controlled", respectively. The solid arrows indicate void volume fractions, including liposome-entrapped suramin; the open arrows indicate fractions containing free suramin. Data are the average of five different liposome preparations.

a cytotoxicity $\left(\mathrm{CC}_{50}\right.$ value of $>6.4 \mu \mathrm{M}$, resulting in a selectivity index $(\mathrm{SI})$ of $>21.4$, which is a promising value for an early formulation screen. As for other formulations, such as suramin lysine salt delivered by polymeric micelles, we could not observe any relevant antiviral activity $\left(\mathrm{EC}_{50}>0.8 \mu \mathrm{M}\right.$ for all formulations, except for suramin lysine salt liposomes, as indicated in Table 3), with a proportionally elevated toxicity, rendering these formulations not adequate for further development. In the case of an $\mathrm{EC}_{50}$ value of $>0.8$, we did not test higher concentrations, as we considered such formulations not worth further investigation, at present, in comparison with the liposomes, where the $\mathrm{EC}_{50}$ value was $0.3 \mu \mathrm{m}$

\section{Conclusions}

Suramin is a highly charged antiviral drug characterized by very low membrane permeability and limited cell internalization. The use of lipophilic carriers, such as liposomes, could enhance the cellular uptake and delivery to the target cells. In the study presented herein, suramin-cationic liposome complexes were prepared by a controlled ethanol injection method, allowing a precise control of ethanol diffusion in water. The resulting liposomes were small, unilamellar and displayed a high encapsulation yield ( $>80 \%$ ). As a result, suramin 
Table 3. Effect of suramin on viral RNA formation in murine norovirus (mNV)-infected cell cultures and on host-cell metabolism.

\begin{tabular}{|lccccc|}
\hline Formulation & \multicolumn{2}{c}{$\mathrm{EC}_{50}[\mu \mathrm{M}]^{[\mathrm{ad}]}$} & $\mathrm{CC}_{50}[\mu \mathrm{M}]^{[\mathrm{b}]}$ & $\mathrm{SI}^{[\mathrm{c}]}$ \\
\hline Suramin lysine salt & $>0.8$ & $>0.8$ & 30.8 & 29.5 & $<37.6$ \\
Suramin lysine salt liposomes (PC 3 mM, DDAB 1 mM) & 0.3 & 0.3 & $>6.4$ & $>6.4$ & $>21.3$ \\
Suramin lysine salt 31R1 polymeric micelles & $>1$ & $>1$ & 0.15 & 0.15 & $<0.15$ \\
Suramin lysine salt P123 polymeric micelles & $>0.8$ & $>0.8$ & $<8$ & $<8$ & $<10$ \\
\hline
\end{tabular}

[a] Compound concentration required to inhibit viral RNA synthesis leading to a decrease in viral load by $50 \%$ in RAW 264.7 macrophages infected with mNV. [b] Compound concentration required to decrease the viability of RAW 264.7 macrophages by $50 \%$. Duplicate cell data shown are the average of three independent experiments; all standard errors were less than $10 \%$. [c] Selectivity index (SI) calculated as the ratio between the $\mathrm{CC}_{50}$ and $\mathrm{EC}_{50}$ values (average of the two experiments).

delivered through liposomes was able to inhibit murine norovirus replication with $\mathrm{EC}_{50}$ values in the micromolar range.

\section{Experimental Section}

Materials: Reagents used for the liposome preparations were phosphatidylcholine (PC) from soybean $(90 \%)$ (phospholipon 90G, Lipoid $\mathrm{GmbH}$, Germany), dimethyl-dioctadecylammonium bromide (DDAB) (Sigma-Aldrich, UK), suramin sodium salt (Sigma-Aldrich, UK), and L-lysine (Sigma-Aldrich, UK). Pluronic P123 and 31R1 were a kind gift from BASF Chem Trade GmbH (Germany). All other reagents were from Sigma-Aldrich (UK) and were of analytical grade. Isotonic palitzsch buffer ( $\mathrm{pH}$ 7.44) was prepared employing biochemical grade components from Sigma-Aldrich (UK). For $100 \mathrm{~mL}$ of buffer solution, $0.05 \mathrm{M}$ sodium tetraborate $(10 \mathrm{~mL})$ were mixed with $0.2 \mathrm{M}$ boric acid $(90 \mathrm{~mL}) ; \mathrm{NaCl}(270 \mathrm{mg})$ was added to adjust the tonicity of the buffer to 0.9 at $37^{\circ} \mathrm{C}$.

Liposome and polymeric micelle preparation: Liposomes were prepared by an ethanol injection method. In brief, $500 \mu \mathrm{L}$ of sura$\min (10 \mathrm{~mm})$ solubilized in a water solution of L-lysine $60 \mathrm{~mm}$ were mixed with $450 \mu \mathrm{L}$ of ethanol/water $(90: 10, v / v)$ solution of PC 30 or $90 \mathrm{~mm}$ plus DDAB $10 \mathrm{~mm}$. A $500 \mu \mathrm{L}$ aliquot of the resulting organic solution was injected by hand or by syringe pump into a $4.4 \mathrm{~mL}$ of double-distilled water under magnetic stirrer for $5 \mathrm{~min}$. Similarly, the preparation of polymeric micelles was accomplished following a procedure described elsewhere, ${ }^{[22]}$ with minor modification. Briefly, ethanol/water $(90: 10, v / v)$ solutions were prepared, containing $30 \mathrm{~mm}$ pluronic $\mathrm{P} 123$ or pluronic $31 \mathrm{R} 1$ plus DDAB $10 \mathrm{~mm}$. Thereafter, $500 \mu \mathrm{L}$ of suramin $(10 \mathrm{~mm})$ solubilized in a $60 \mathrm{~mm}$ aqueous solution of L-lysine were mixed with $450 \mu \mathrm{L}$ of the pluronic solutions. A $500 \mu \mathrm{L}$ aliquot of the resulting pluronic/ $\mathrm{DDAB} /$ suramin solution were injected by syringe pump in a $4.4 \mathrm{~mL}$ of double-distilled water under magnetic stirrer for $5 \mathrm{~min}$. Both the preparation of liposomes and polymeric micelles were performed at room temperature, typically $21-23^{\circ} \mathrm{C}$.

\section{Liposome characterization}

Photon correlation spectroscopy (PCS): Submicron particle size analyses of the liposomes were performed using a Zetasizer 3000 PCS instrument (Malvern Instruments, UK). PCS analysis was performed using a $5 \mathrm{~mW}$ helium neon laser with a wavelength output of $633 \mathrm{~nm}$. Glassware was cleaned of dust by washing with detergent and rinsing twice with water for injections. Measurements were taken at $25^{\circ} \mathrm{C}$ and an angle of $90^{\circ}$. Data were elaborated using the Zetasizer Software 7.03. ${ }^{[17]}$
Cryogenic transmission electron microscopy (cryo-TEM): A $3 \mu \mathrm{L}$ aliquot of sample solution was applied on plasma-treated (Gatan Solarus Model 950 Advanced Plasma System, $\quad p=70$ mTorr, $\mathrm{H}_{2}$ flow $6.4 \mathrm{sccm}, \mathrm{O}_{2}$ flow $27.5 \mathrm{sccm}$, forward RF target $50 \mathrm{~W}$, time $30 \mathrm{~s}$ ) carbon copper grids (Quantifoil R 3.5/1) in the environmental chamber of fully automated vitrification device for plunge freezing (FEI Vibrot), having relative air humidity of $100 \%$ and temperature of $22^{\circ} \mathrm{C}$. The excess solution was removed by blotting with filter paper for $2 \mathrm{~s}$ followed by $1 \mathrm{~s}$ draining and plunging of the samples into 1:1 mixture of liquid ethane and liquid propane, which was cooled to $-170^{\circ} \mathrm{C}$. Vitrified samples were cryo-transferred into Jeol JEM$3200 F S C$ cryo-TEM operating at $-194^{\circ} \mathrm{C}$. The temperature of the samples was $-187^{\circ} \mathrm{C}$ during image acquisition. The microscope was operating in the bright field mode, using $300 \mathrm{kV}$ acceleration voltage; the in-column energy filter was set to $0-20 \mathrm{eV}$ energy-loss range (zero-loss imaging). Micrographs were recorded with a Gatan Ultrascan 4000 CCD camera.

Atomic force microscopy (AFM): AFM images were obtained with a Nanoscope III (Digital Instruments, Santa Barbara, CA, USA), equipped with single silicon crystal cantilever, in tapping mode at room temperature. Droplets of $10 \mu \mathrm{L}$ constant volume were deposited on freshly cleaved mica, for $15 \mathrm{~min}$, without any dilution of the sample. The scanning area was $2 \mu \mathrm{m}^{2}$, and the scan rate was around $1.0 \mathrm{~Hz}$. The force between tip and sample (set point) was carefully controlled and adjusted in order to prevent destroying the liposome structure.

Determination of encapsulation efficiency: A gel filtration method was established to separate the suramin-loaded liposomes. The encapsulation efficiency was determined by using an UV spectrophotometer. Liposomes were loaded into a sepharose $4 B$ column (diameter: $1.5 \mathrm{~cm}$, length: $50 \mathrm{~cm}$, Pharmacia, Uppsala, Sweden) pre-equilibrated and eluted with borate buffer. The void volume peak fractions containing the suramin-loaded liposomes were collected and quantitated for liposome and suramin content. The UV-Vis spectrum of each fraction was measured both in isotonic palitzsch buffer and in ethanol/water (70:30) in order to estimate the turbidity of the suspension (optical density at $600 \mathrm{~nm}$, when measured in buffer), and in order to determine the concentration of suramin (optical density at $318 \mathrm{~nm}$, when measured in ethanol/ water). All of the UV spectra were recorded on a Hewlett-Packard 8452 diode array spectrophotometer. The total and free amounts of suramin resulting ( $C_{0}$ and $C_{1}$, respectively), were used to calculate the encapsulation efficiency (EE) according to Equation (1).

$\mathrm{EE}=\left(1-C_{1} / C_{0}\right) \times 100 \%$

Viruses and cells: Murine norovirus strain was passaged once in RAW 264.7 macrophages to prepare a working virus stock, and the cell cultures were stored at $-80^{\circ} \mathrm{C}$ until further use.

Cytotoxic and antiviral cell-based assays: To assess the toxicity and antiviral potency of the formulated suramin-loaded liposomes and micelles in RAW 264.7 macrophages, a cell proliferation assay was performed as previously described. Briefly, RAW 264.7 macrophages were seeded into a 96 -well plate with $7.5 \times 10^{4}$ cells/well. A 
serial dilution of formulated suramin-loaded liposomes or micelles, at a concentration ranging from $50 \mu \mathrm{M}$ to $0.125 \mu \mathrm{M}$ or from $0.8 \mu \mathrm{M}$ to $0.125 \mu \mathrm{M}$, was added to the non-infected or mNV-infected cells, respectively. After $24 \mathrm{~h}, 100 \mu \mathrm{L}$ of 3-(4,5-dimethylthiazol-2-yl)-5-(3carboxymethoxyphenyl)-2-(4-sulfophenyl)- $2 \mathrm{H}$-tetrazolium (MTS) solution were added to each well. After $2 \mathrm{~h}$, absorbance of the solution was measured at $490 \mathrm{~nm}$, and the $\mathrm{CC}_{50}$ and $\mathrm{EC}_{50}$ values determined. ${ }^{[23]}$

\section{Acknowledgements}

This work was funded by the European Union's Seventh Framework Programme (FP7) HEALTH-2010 Collaborative Project SILVER (no. 260644)

Keywords: antiviral agents · drug delivery • liposomes . norovirus - RNA-dependent RNA polymerase

[1] M. M. Patel, M. A. Widdowson, R. I. Glass, K. Akazawa, J. Vinje, U. D. Parashar, Emerging Infect. Dis. 2008, 14, 1224-1231.

[2] R. M. Turcios-Ruiz, P. Axelrod, K. St John, E. Bullitt, J. Donahue, N. Robinson, H. E. Friss, J. Pediatr. 2008, 153, 339-344.

[3] S. Y. Chen, C. N. Tsai, M. W. Lai, C. Y. Chen, K. L. Lin, T. Y. Lin, C. H. Chiu, Clin. Infect. Dis. 2009, 48, 849-855.

[4] M. P. Girard, D. Steele, C. L. Chaignat, M. P. Kieny, Vaccine 2006, 24, $2732-2750$.

[5] I. N. Clarke, P. R. Lambden, J. Infect. Dis. 2000, 181, S309-316.

[6] S. W. Fullerton, M. Blaschke, B. Coutard, J. Gebhardt, A. Gorbalenya, B. Canard, P. A. Tucker, J. Rohayem, J. Virol. 2007, 81, 1858-1871.

[7] E. Mastrangelo, M. Pezzullo, D. Tarantino, R. Petazzi, F. Germani, D. Kramer, I. Robel, J. Rohayem, M. Bolognesi, M. Milani, J. Mol. Biol. 2012 $419,198-210$
[8] F. Hawking, Adv. Pharmacol. Chemother. 1978, 15, 289-322.

[9] M. Kaur, E. Reed, O. Sartor, W. Dahut, W. D. Figg, Invest. New Drugs 2002, 20, 209-219.

[10] L. D. Kaplan, P. R. Wolfe, P. A. Volberding, P. Feorino, J. A. Levy, D. I. Abrams, D. Kiprov, R. Wong, L. Kaufman, M. S. Gottlieb, Am. J. Med. $1987,82,615-620$

[11] S. A. Grossman, S. Phuphanich, G. Lesser, J. Rozental, L. B. Grochow, J. Fisher, S. Piantadosi; New Approaches to Brain Tumor Therapy CNS Consortium, J. Clin. Oncol. 2001, 19, 3260-3266.

[12] S. S. Chrai, R. Murari, A. Imran, BioPharm 2001, 14, 10-14.

[13] K. Motoyama, Y. Nakashima, Y. Aramaki, F. Hirayama, K. Uekama, H. Arima, J. Drug Delivery 2011, 2011, 476137.

[14] J. Lin, Y. Yu, S. Shigdar, D. Z. Fang, J. R. Du, M. Q. Wei, A. Danks, K. Liu, W. Duan, PloS One 2012, 7, e49277.

[15] M. S. P. S. S. Patel, S. Salampure, B. Vishwanath, N. M. Patel, J. Sci. Res. 2010, 2, 585-596.

[16] J. Song, F. Shi, Z. Zhang, F. Zhu, J. Xue, X. Tan, L. Zhang, X. Jia, Molecules 2011, 16, 7880-7892.

[17] R. Cortesi, E. Esposito, S. Gambarin, P. Telloli, E. Menegatti, C. Nastruzzi, J. Microencapsulation 1999, 16, 251-256.

[18] C. Mugabe, A. O. Azghani, A. Omri, Int. J. Pharm. 2006, 307, 244-250.

[19] P. Stano, S. Bufali, C. Pisano, F. Bucci, M. Barbarino, M. Santaniello, P. Carminati, P. L. Luisi, J. Liposome Res. 2004, 14, 87-109.

[20] C. Jaafar-Maalej, R. Diab, V. Andrieu, A. Elaissari, H. Fessi, J. Liposome Res. 2010, 20, 228-243.

[21] J. Rohayem, Clin. Microbiol. Infect. 2009, 15, 524-527.

[22] L. Capretto, S. Mazzitelli, E. Brognara, I. Lampronti, D. Carugo, M. Hill, X. Zhang, R. Gambari, C. Nastruzzi, Int. J. Nanomed. 2012, 7, 307-324.

[23] J. Rohayem, M. Bergmann, J. Gebhardt, E. Gould, P. Tucker, A. Mattevi, T. Unge, R. Hilgenfeld, J. Neyts, Antiviral Res. 2010, 87, 162-178.

Received: December 28, 2013

Published online on March 11, 2014 